\title{
Compuestos ablativos de matriz polimérica (resina poliéster) reforzada con materiales de desecho industrial y fibra de vidrio*
}

\author{
Julián Portocarrero Hermann** \\ John Darío Osorio *** \\ Rafael Andres Robayo-Salazar ${ }^{* * * *}$
}

\author{
Recibido: 18/06/2019 • Aceptado: 16/09/2019 \\ https://doi.org/10.22395/rium.v19n36a11
}

\begin{abstract}
Resumen
Los materiales ablativos son la base de los sistemas de protección térmica de equipos o elementos recuperables que, en servicio, son expuestos a elevadas temperaturas. Generalmente, estos materiales son de difícil adquisición y alto costo, lo que dificulta su aplicación en países como Colombia. Esta investigación describe la obtención y caracterización de materiales compuestos de tipo ablativo basados en una matriz de resina poliéster reforzada con materiales particulados (60-70 \% en peso) y fibra de vidrio corta (5\% en peso). Como particulados, se utilizaron residuos cerámicos y escoria de aluminio finamente molidos $(<75 \mu \mathrm{m})$, destacando que ambos materiales son subproductos industriales. Los compuestos desarrollados fueron sometidos al ensayo de llama directa con el fin de evaluar su desempeño ablativo. De esta manera, se simularon las condiciones térmicas $\left(1.600-2.000{ }^{\circ} \mathrm{C}\right)$ a las cuales se expone la tobera de un cohete de órbita baja (tipo sonda). Complementariamente, se evaluó el efecto del ensayo ablativo sobre el nivel de agrietamiento y erosión (pérdida de peso) de los compuestos desarrollados. Como medida indirecta del desempeño mecánico residual de los compuestos, fue determinada la dureza de la zona térmicamente afectada. Los resultados obtenidos son considerados de suma importancia para la producción de sistemas de protección térmica o componentes de propulsión con materias primas de fácil adquisición y bajo costo en Colombia.
\end{abstract}

Palabras clave: materiales compuestos; materiales ablativos; sistemas de protección térmica; ensayo de llama directa; industria aeroespacial.

Articulo original derivado de una investigación en curso, apoyada por el Grupo de Investigación en Estudios Aeroespaciales (GIEA) de la Escuela Militar de Aviación Marco Fidel Suarez (Emavi) y la Fuerza Aérea Colombiana (FAC).

** Ingeniero mecánico. Profesor, integrante del Grupo de Investigación en Estudios Aeroespaciales (GIEA), Programa de Ingeniería Mecánica (Pimec), Escuela Militar de Aviación (Emavi), Cali, Colombia. Correo electrónico: julian.portocarrero@emavi.edu.co. Orcid: http://orcid.org/0000-0002-4573-1435

*** Ingeniero mecánico. Integrante del Grupo de Investigación en Estudios Aeroespaciales (GIEA), Programa de Ingeniería Mecánica (Pimec), Escuela Militar de Aviación (Emavi), Cali, Colombia. Correo electrónico: jhon. osorio@fac.mil.co. Orcid: http://orcid.org/0000-0002-8397-7559

**** Doctor en Ingeniería. Profesor investigador, integrante del Grupo de Investigación en Estudios Aeroespaciales (GIEA), Programa de Ingeniería Mecánica (Pimec), Escuela Militar de Aviación (Emavi), Cali, Colombia. Correo electrónico: rafael.robayo@emavi.edu.co. Orcid: https://orcid.org/0000-0003-1687-2885 


\title{
Ablative Composites of Polymeric Matrix (Polyester Resin) Reinforced With Industrial Residue Materials and Fiberglass
}

\begin{abstract}
The ablative materials are the base of the thermal protection systems for equipment and recoverable elements that, when used, are exposed to high temperatures. Generally, these materials are hard to acquire and costly, which makes difficult their application in countries such as Colombia. This research describes the acquisition and characterization of ablative composites based on a matrix of polyester resin reinforced with particulate materials (60-70\% in weight) and fiberglass (5\% in weight). Ceramic residues and aluminum dross finely ground $(<75 \mu \mathrm{m})$ were used as particulates, highlighting that both materials are industrial byproducts. The developed composites were put through the direct flame test to assess their ablative performance. Thus, the thermal conditions $\left(1,600-2,000{ }^{\circ} \mathrm{C}\right)$ to which the nozzle of a low orbit rocket (prove type) is exposed were simulated. Complementarily, the effect of the ablative test over the cracking level and erosion (loss of weight) of the developed composites were assessed. As an indirect measure of the residual mechanic performance of the composites the hardness of the thermally affected area was determined. The gathered results are considered highly important for the production of thermic protection systems or propelling composites with raw materials of easier acquisition and lower cost in Colombia.
\end{abstract}

Keywords: composite materials; ablative materials; thermal protective systems; direct flame test; aerospace industry.

\section{Compósitos ablativos de matriz polimérica (resina poliéster) reforçada com materiais de resíduo industrial e fibra de vidro}

\begin{abstract}
Resumo
Os materiais ablativos são a base dos sistemas de proteção térmica de equipamentos ou elementos recuperáveis que, em serviço, são expostos a elevadas temperaturas. Em geral, esses materiais são de difícil aquisição e de alto custo, o que dificulta sua aplicação em países como a Colômbia. Esta pesquisa descreve a obtenção e a caracterização de materiais compostos de tipo ablativo baseados em uma matriz de resina poliéster reforçada com materiais particulados (60-70\% em peso) e fibra de vidro curta ( 5 \% em peso). Como particulados, foram utilizados resíduos cerâmicos e escória de alumínio finamente moídos $(<75 \mu \mathrm{m})$, destacando que ambos os materiais são subprodutos industriais. Os compósitos desenvolvidos foram submetidos ao ensaio de chama direta com o objetivo de avaliar seu desempenho ablativo. Dessa maneira, foram estimadas as condições térmicas $\left(1.600-2.000{ }^{\circ} \mathrm{C}\right)$ às quais o bocal de um foguete de órbita baixa (tipo sonda) foi exposto. Além disso, foi avaliado o efeito do ensaio ablativo sobre o nível de rachadura e erosão (perda de peso) dos componentes desenvolvidos. Como medida indireta do desempenho mecânico residual dos compósitos, foi determinada a dureza da zona termicamente afetada. Os resultados obtidos são considerados de grande relevância para a produção de sistemas de proteção térmica ou componentes de propulsão com matérias-primas de fácil aquisição e de baixo custo na Colômbia.
\end{abstract}

Palavras-chave: materiais compostos; materiais ablativos; sistemas de proteção térmica; ensaio de chama direta; indústria aeroespacial. 


\section{INTRODUCCIÓN}

Los materiales ablativos se reconocen como materiales fungibles (de sacrificio), cuyas propiedades permiten catalogarlos como sistemas de protección térmica (TPS) en elementos recuperables que, en servicio, son expuestos a elevadas temperaturas $(\geq 1.500$ $\left.{ }^{\circ} \mathrm{C}\right)$ [1]. Las funciones de un material ablativo se resumen en el hecho de ser refractario, aislante térmico, resistente al desgaste, autoconsumible y resistente en cuanto a los esfuerzos térmicos [2]. Los campos de aplicación más relevantes para los TPS son las industrias automovilística, aeronáutica y aeroespacial. En relación con esta última, se destacan aplicaciones como la protección de naves espaciales y satélites durante el vuelo hipersónico a través de la atmósfera planetaria. Asimismo, se resalta la fabricación de elementos de propulsión como las toberas de motor, entre otras [3]. Generalmente, los TPS se reconocen por su elevado costo y difícil adquisición, en gran parte debido a los materiales ablativos comúnmente utilizados y la tecnología involucrada en sus procesos de fabricación, lo cual es considerado como una de las principales limitantes para el desarrollo de estas industrias a nivel regional.

Dentro de los materiales actualmente utilizados para la fabricación de TPS, se destacan los compuestos ablativos de matriz polimérica (PACMs), debido a su versatilidad y la posibilidad de reducir el peso $(\approx 90 \%)$ de los componentes sin sacrificar las propiedades ablativas de interés [4]. De hecho, en comparación con los materiales ablativos no-poliméricos, tales como los metales de alto punto de fusión (tungsteno, renio y molibdeno), compuestos cerámicos refractarios (carburos de silicio ( $\mathrm{SiC}$ ), circonio (ZrC), boro (B4C) o hafnio (HfC)) y polímeros inorgánicos; los PACMs tienen algunas ventajas intrínsecas como su densidad ajustable, bajo costo, menor conductividad térmica, elevada resistencia a la corrosión, mayor resistencia al choque térmico y la posibilidad de descomponerse térmicamente en productos volátiles de bajo peso molecular, entre otras [5-6].

Por lo general, las resinas poliméricas (fenólicas, epoxi, poliéster y viniléster) utilizadas como aglomerante en los PACMs son termoestables y su desempeño a elevadas temperaturas es limitado [7]. Por esta razón, son modificadas con la incorporación de refuerzos particulados (rellenos) de tipo cerámico u óxidos refractarios (hafnio $\left(\mathrm{HfO}_{2}\right)$, circonio $\left(\mathrm{ZrO}_{2}\right)$, tántalo $\left(\mathrm{Ta}_{2} \mathrm{O}_{5}\right)$, itrio $\left(\mathrm{Y}_{2} \mathrm{O}_{3}\right)$ o alúmina $\left(\mathrm{Al}_{2} \mathrm{O}_{3}\right)$ ), y al mismo tiempo reforzadas con fibras cortas de tipo carbono, asbestos o vidrio [8-11]. Cuando los PACMs son expuestos a temperaturas por encima del límite de degradación térmica de la matriz (resina), su estructura experimenta descomposición (pirólisis) y se produce una capa carbonizada (figura 1) o un residuo debido al carbono presente en la estructura orgánica de la resina; al respecto, el carbono es considerado un material ablativo de alto rendimiento. Esta capa carbonizada promueve la retención parcial (aglomeración) 
de los rellenos incorporados y el proceso de ablación se puede llevar a cabo de manera efectiva, ya que el material puede continuar absorbiendo calor endotérmicamente [12]. Aun considerando las propiedades ablativas de esta capa, el residuo carbonizado y los rellenos pueden erosionarse mecánicamente (delaminación) o eliminarse por fricción, afectando el desempeño y rendimiento del TPS. Para este propósito, las fibras cortas se han incorporado con éxito, logrando mantener la integridad del compuesto ablativo, aumentando su resistencia a la erosión y a los esfuerzos térmicos $[5,13]$.

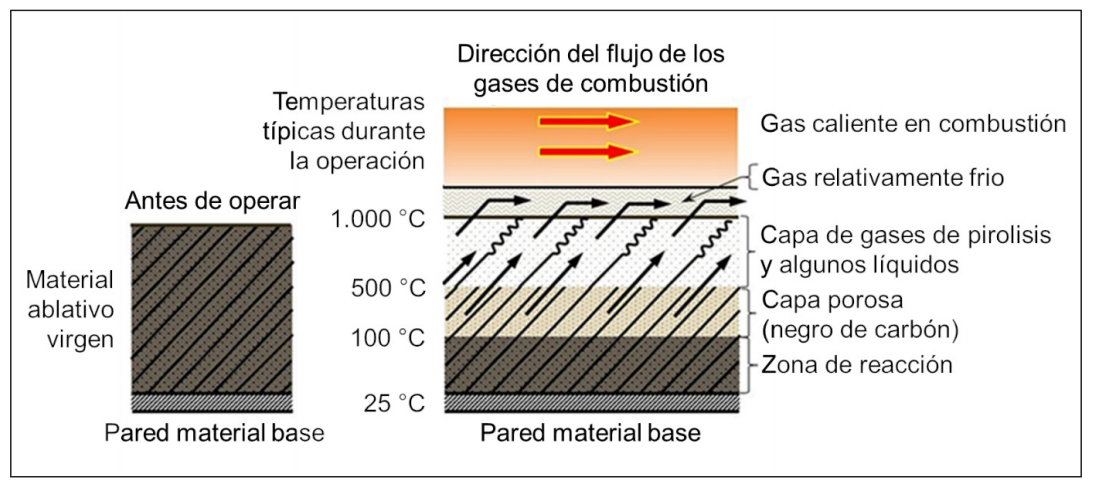

Figura 1. Representacion gráfica de las zonas formadas durante la exposición a altas temperaturas de un sistema de protección térmica

Fuente: elaboración propia basada en [16].

Para el sector aeroespacial y aeronáutico colombiano, es una necesidad fabricar TPS con materiales de fácil adquisición y disponibilidad local que le permitan su desarrollo con poca dependencia tecnológica externa. Con este propósito, este artículo de investigación describe la obtención de materiales compuestos con propiedades ablativas basados en una matriz polimérica (resina poliéster) modificada con materiales particulados y reforzada con fibra de vidrio. Se evaluó el efecto del contenido y tipo de material particulado (polvo cerámico y escoria de aluminio) sobre el desempeño ablativo (llama directa) de los compuestos, destacando que los refuerzos particulados utilizados son residuos o subproductos de la industria local. Complementariamente, se evaluó la dureza de la zona afectada térmicamente, además del efecto de la incorporación de fibra de vidrio sobre el control de agrietamiento y pérdida de peso (erosión) de los compuestos bajo las condiciones de servicio simuladas (temperaturas entre $1.600 \mathrm{y}$ $2.000{ }^{\circ} \mathrm{C}$ ). Los resultados obtenidos se consideran de suma importancia para la producción local de sistemas de protección térmica y su aplicación real, en este caso, en la manufactura de componentes de propulsión como las toberas de cohetes de órbita baja (tipo sonda) que la Fuerza Aérea Colombiana (FAC) pretende desarrollar para fines científicos, tecnológicos y militares, con poca dependencia tecnológica externa. 


\section{MATERIALES Y METODOLOGÍA}

Como materias primas para la obtención del compuesto ablativo, se utilizó una matriz de resina poliéster insaturada y preacelerada (NovaSuin P436 Ultra) y dos tipos de refuerzo: particulados y fibra de vidrio (F) tipo E. Los particulados corresponden a polvo cerámico (C) y escoria de aluminio (E), subproductos de las industrias de cerámica y de fundición de aluminio, respectivamente. Ambos materiales fueron molturados hasta lograr un tamaño de partícula menor a las $75 \mu \mathrm{m}$. Los porcentajes de incorporación de los particulados fueron entre $60-70 \%$ en peso respecto a la mezcla de material compuesto. La fibra de vidrio fue cortada a una longitud de $4 \pm 1 \mathrm{~mm}$ y fue incorporada en un porcentaje del $5 \%$ respecto al peso de la mezcla de material compuesto.

Con base en las dosificaciones de la tabla 1 se elaboraron los PACMs. Durante el mezclado, la resina poliéster fue catalizada con la adición de un $1 \%$ (respecto al peso de la resina) de PMEC (peróxido de metil-etil-cetona). Las mezclas fueron vertidas en moldes de 19,5 x 13,5 x 1,0 cm (largo, ancho y espesor), los cuales posteriormente fueron vibrados y sometidos a un proceso de prensado con el fin de reducir las burbujas de aire atrapadas durante el mezclado y moldeo. El prensado de las placas se realizó en una prensa hidráulica manual (Jack $6 \mathrm{t}$ ) y se mantuvo hasta garantizar el endurecimiento de las mezclas $(5 \mathrm{~h})$ y un espesor final de las placas entre 7-8 $\mathrm{mm}$. El nivel de carga aplicada variaba hasta lograr dicho espesor. La figura 2 resume de manera esquemática la metodología seguida para la obtención de las placas del material compuesto.

Tabla 1. Composición de las mezclas desarrolladas para la elaboración de las placas de material compuesto

\begin{tabular}{|c|c|c|c|c|}
\hline \multirow{2}{*}{$\begin{array}{l}\text { Referencia } \\
\text { Compuesto }\end{array}$} & \multirow{2}{*}{$\begin{array}{l}\text { Tipo de refuerzo } \\
\text { particulado }\end{array}$} & $\begin{array}{c}\text { Contenido de refuerzo } \\
\text { particulado }\end{array}$ & $\begin{array}{l}\text { Contenido de refuerzo } \\
\text { de fibra de vidrio }(F)\end{array}$ & $\begin{array}{c}\text { Contenido de resina } \\
\text { poliéster }\end{array}$ \\
\hline & & \multicolumn{3}{|c|}{ (\% en peso) } \\
\hline $60 \mathrm{C}$ & \multirow{3}{*}{ Cerámico $(\mathrm{C})$} & 60 & \multirow{2}{*}{0} & 40 \\
\hline $70 \mathrm{C}$ & & 70 & & 30 \\
\hline $65 \mathrm{C}-5 \mathrm{~F}$ & & 65 & 5 & 30 \\
\hline $60 \mathrm{E}$ & \multirow{3}{*}{ Escoria (E) } & 60 & \multirow{2}{*}{0} & 40 \\
\hline $70 \mathrm{E}$ & & 70 & & 30 \\
\hline $65 \mathrm{E}+5 \mathrm{~F}$ & & 65 & 5 & 30 \\
\hline $30 \mathrm{C}+30 \mathrm{E}$ & \multirow{3}{*}{$\begin{array}{l}\text { Cerámico }(\mathrm{C}) \\
+ \text { Escoria }(\mathrm{E})\end{array}$} & $30 \mathrm{C}+30 \mathrm{E}$ & \multirow{2}{*}{0} & 40 \\
\hline $35 \mathrm{C}+35 \mathrm{E}$ & & $35 \mathrm{C}+35 \mathrm{E}$ & & 30 \\
\hline $\begin{array}{l}32,5 \mathrm{C}+ \\
32,5 \mathrm{E}+5 \mathrm{~F}\end{array}$ & & $32,5 \mathrm{C}+32,5 \mathrm{E}+5 \mathrm{~F}$ & 5 & 30 \\
\hline
\end{tabular}

Fuente: elaboración propia 
a)

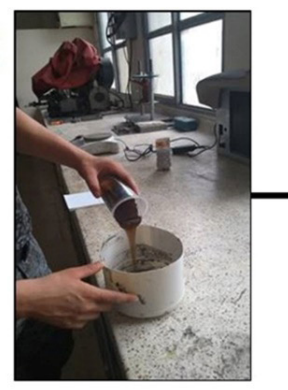

d)

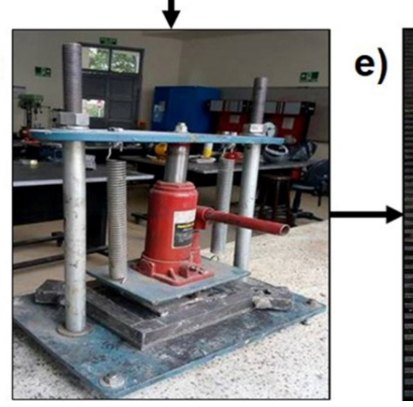

b)

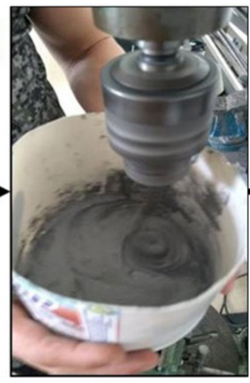

c)
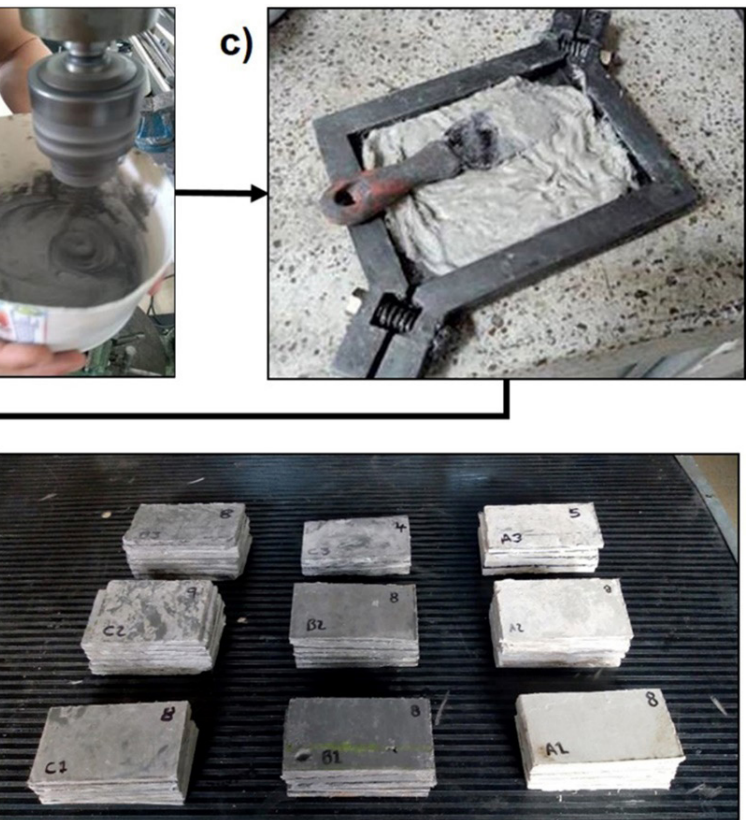

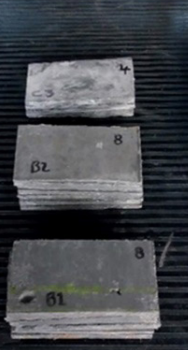

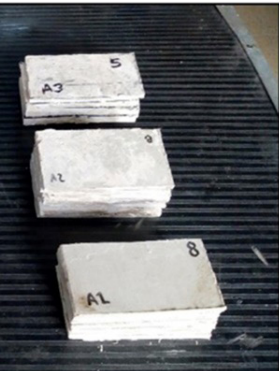

Figura 2. Metodología seguida para la fabricación de las placas: a) dosificación de las materias primas, b) proceso de mezclado con la adaptación de un taladro de árbol vertical, c) moldeo de las placas, d) proceso de prensado, y e) placas elaboradas

Fuente: elaboración propia

Las placas de material compuesto elaboradas (ocho por mezcla) fueron sometidas al ensayo de llama directa $\left(1.600-2.000^{\circ} \mathrm{C}\right)$ durante ciento veinte segundos con el fin de evaluar su desempeño como materiales ablativos, adaptando el procedimiento descrito en la norma ASTM E285 [14]. Este rango de temperaturas simula la condición térmica de operación de la tobera de propulsión de un cohete de órbita baja (tipo sonda). Sin embargo, el ensayo de llama directa se considera más severo, dado que la incidencia de la llama es frontal o perpendicular a la placa, a diferencia de la incidencia tangencial que ocurre durante la propulsión (figura 1). Como fuente de la llama se utilizó un soplete (antorcha) de gas propano, el cual se fijó a una distancia de $4 \mathrm{~cm}$ frente a cada placa de ensayo (figura 3a). El comportamiento aislante fue evaluado mediante el monitoreo del perfil de temperatura de las placas por medio de una cámara termográfica FLIR E4, que registra la temperatura de la llama incidente, y un termopar adherido a la zona posterior de la cara expuesta a la llama (figura 3b). El efecto de la incorporación de fibra de vidrio sobre el control de agrietamiento y la delaminación de la zona afectada térmicamente fue evaluado mediante la inspección visual en un estereomicroscopio trinocular Advance Optical SQF-E. Los hallazgos fueron relacionados con la pérdida de peso (erosión térmica) y la dureza de las placas al terminar el ensayo de ablación térmica. 
La determinación de la dureza se llevó a cabo siguiendo el procedimiento descrito en la norma ASTM D2583 [15] utilizando un durómetro portátil Barcol Impressor.

a)
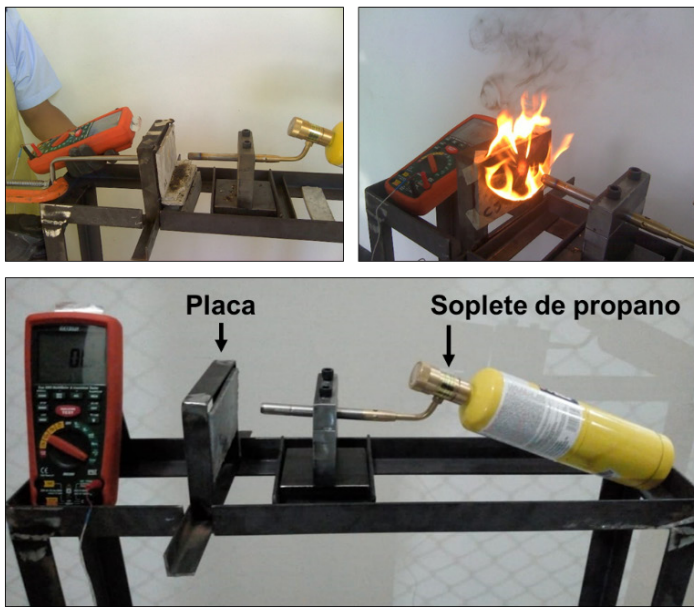

b)
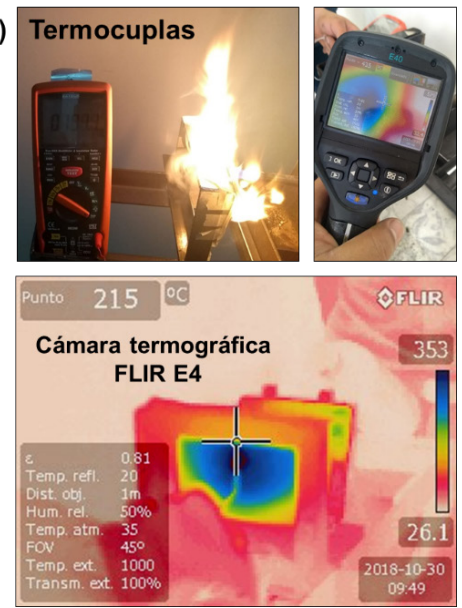

Figura 3. a) Montaje del ensayo de llama directa. b) Determinación del gradiente de temperatura por medio de cámara termográfica (llama incidente) y termocupla (cara posterior a la zona térmicamente afectada)

Fuente: elaboración propia

\section{RESULTADOS Y ANÁLISIS}

\subsection{Ensayo ablativo (llama directa)}

Los resultados del ensayo ablativo se pueden observar en la tabla 2.

Tabla 2. Resultados del ensayo ablativo (llama directa)

\begin{tabular}{|c|c|c|c|c|}
\hline $\begin{array}{l}\text { Referencia } \\
\text { Compuesto }\end{array}$ & $\begin{array}{l}\text { Espesor de las } \\
\text { placas }(\mathrm{mm})\end{array}$ & $\begin{array}{l}\text { Temperatura } \\
\text { incidente }\left({ }^{\circ} \mathrm{C}\right)\end{array}$ & $\begin{array}{c}\text { Temperatura cara } \\
\text { opuesta a la llama }\left({ }^{\circ} \mathrm{C}\right)\end{array}$ & $\begin{array}{c}\text { Relación } \\
\Delta T / \text { Espesor }\left({ }^{\circ} \mathrm{C} / \mathrm{mm}\right)\end{array}$ \\
\hline $60 \mathrm{C}$ & $7,3 \pm 0,7$ & \multirow{9}{*}{1.600} & $226,6 \pm 28,3$ & $189,0 \pm 18,2$ \\
\hline $70 \mathrm{C}$ & $7,0 \pm 0,4$ & & $209,7 \pm 32,8$ & $199,2 \pm 7,3$ \\
\hline $65 \mathrm{C}+5 \mathrm{~F}$ & $8,2 \pm 0,7$ & & $202,8 \pm 27,3$ & $172,5 \pm 17,1$ \\
\hline $60 \mathrm{E}$ & $7,5 \pm 0,8$ & & $291,8 \pm 48,1$ & $177,6 \pm 23,1$ \\
\hline $70 \mathrm{E}$ & $6,9 \pm 0,8$ & & $251,6 \pm 47,0$ & $197,8 \pm 27,8$ \\
\hline $65 \mathrm{E}+5 \mathrm{~F}$ & $7,2 \pm 0,6$ & & $206,9 \pm 43,9$ & $195,3 \pm 14,8$ \\
\hline $30 \mathrm{C}+30 \mathrm{E}$ & $7,2 \pm 0,6$ & & $247,8 \pm 25,4$ & $188,8 \pm 16,1$ \\
\hline $35 \mathrm{C}+35 \mathrm{E}$ & $6,9 \pm 0,6$ & & $236,6 \pm 35,8$ & $199,7 \pm 15,6$ \\
\hline $32,5 \mathrm{C}+32,5 \mathrm{E}+5 \mathrm{~F}$ & $7,0 \pm 0,5$ & & $225,2 \pm 43,7$ & $195,8 \pm 13,0$ \\
\hline
\end{tabular}

Fuente: elaboración propia 
En general, se destaca cómo la temperatura de la cara opuesta a la llama directa presentó un rango entre $202-292{ }^{\circ} \mathrm{C}$, lo que significa una reducción de la temperatura incidente $\left(1.600^{\circ} \mathrm{C}\right)$ de hasta el $87 \%$, demostrando la capacidad aislante de los PACMs desarrollados. Al comparar las temperaturas de la cara opuesta a la llama entre los PACMs reforzados con polvo cerámico (C) y escoria de aluminio (E), se puede deducir que los materiales ablativos desarrollados con polvo cerámico presentan la mayor capacidad aislante, al reportar las temperaturas más bajas. Este hecho puede estar relacionado con las diferencias en conductividad térmica entre ambos tipos de materiales o alguna reacción endotérmica producida en el cerámico que promueve un mayor grado de absorción de calor. Por su parte, el aumento del contenido de material particulado ( $60 \%$ vs. $70 \%$ ) promovió un mayor nivel de aislamiento térmico, siendo este efecto más notorio para los PACMs reforzados con escoria de aluminio. En efecto, el compuesto 70E presentó una temperatura de la cara opuesta un 13,8 \% menor que el compuesto 60E. A manera comparativa, el compuesto $70 \mathrm{C}$ logró una reducción del 7,5\% con relación al valor reportado por el compuesto $60 \mathrm{C}$. Asimismo, la incorporación de fibra de vidrio (F) (5\%) logró incrementar la capacidad aislante de los PACMs. De igual manera fue el caso del compuesto $65 \mathrm{E}+5 \mathrm{~F}$, que reportó una temperatura de la cara opuesta a la llama $44,7^{\circ} \mathrm{C}$ menor respecto a la reportada por el compuesto $70 \mathrm{E}\left(206,9^{\circ} \mathrm{C}\right.$ vs. $\left.251,6^{\circ} \mathrm{C}\right)$. La relación entre el cambio $(\Delta \mathrm{T})$ de temperatura $\left({ }^{\circ} \mathrm{C}\right)$ y el espesor de cada placa $(\mathrm{mm})$ se puede apreciar en la tabla 2. En general, esta relación corrobora que el aumento del contenido de material particulado (60 \% vs. $70 \%)$ promueve un mayor comportamiento aislante en los compuestos.

\subsection{Pérdida de peso (erosión térmica)}

La tasa de erosión, en términos de pérdida de masa de los PACMs desarrollados, se puede observar en la figura 4.

En general, se evidencia que el incremento del contenido de material particulado (60\% vs. $70 \%$ ) disminuye la tasa de erosión (pérdida de peso) durante la prueba de ablación, lo cual indica que las partículas actúan reduciendo el área afectada por la llama. Este hallazgo coincide con los resultados reportados por Asaro et al. [9] y Krzyzak et al. [8], quienes utilizaron partículas mesoporosas de sílice, negro de carbón (5-20\% en peso) y perlita expandida (2-32\% en volumen) como estrategias para mejorar el comportamiento ablativo de resinas fenólicas y epóxicas. Por su parte, la incorporación de fibra de vidrio (F) (5\%) resultó en una reducción de la erosión de los PACMs de hasta el 64,4\%. Estos resultados indican que, en una aplicación real, la incorporación conjunta del $5 \%$ de fibra de vidrio y $65 \%$ de material particulado (polvo cerámico y/o escoria de aluminio) conduce a mayores tiempos de protección, lo cual se traduce en una ventaja de gran importancia tecnológica e industrial. El mejor 
desempeño fue el mostrado por el compuesto $65 \mathrm{E}+5 \mathrm{~F}$, con una pérdida de peso de tan solo el 2,0 \% durante los $120 \mathrm{~s}$ de ensayo.

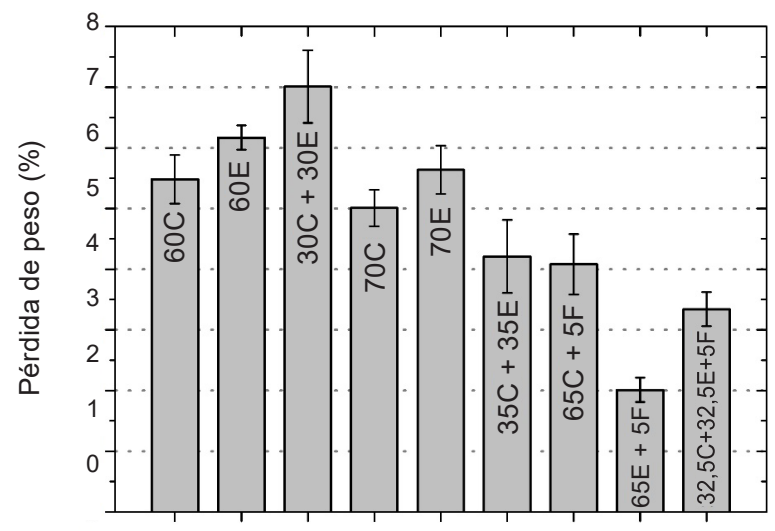

Tipo de compuesto

Figura 4. Pérdida de peso de las placas sometidas al ensayo ablativo (llama directa)

Fuente: elaboración propia

\subsection{Ensayo de dureza Barcol}

Como medida indirecta del desempeño mecánico residual de los PACMs, posterior al ensayo de llama directa, fue determinada la dureza de la zona térmicamente afectada y esta fue comparada con la dureza de la zona adyacente o no afectada por la llama incidente. La dureza (Barcol) de ambas zonas para los PACMs desarrollados se puede observar en la figura 5 .

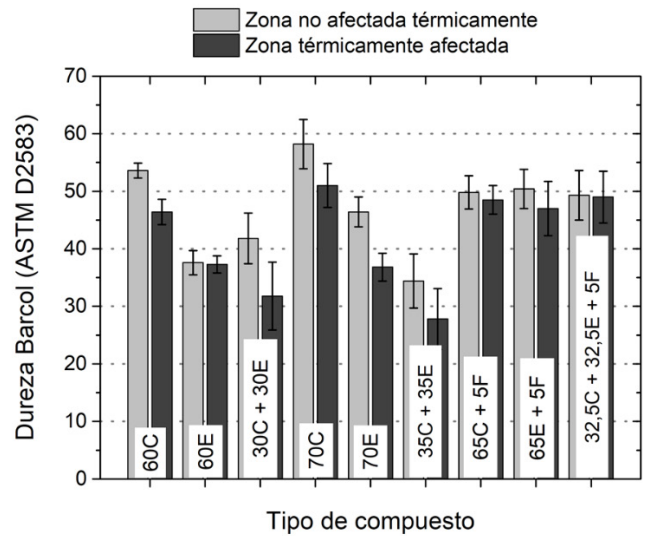

Ensayo de dureza Barcol (ASTM D2583)

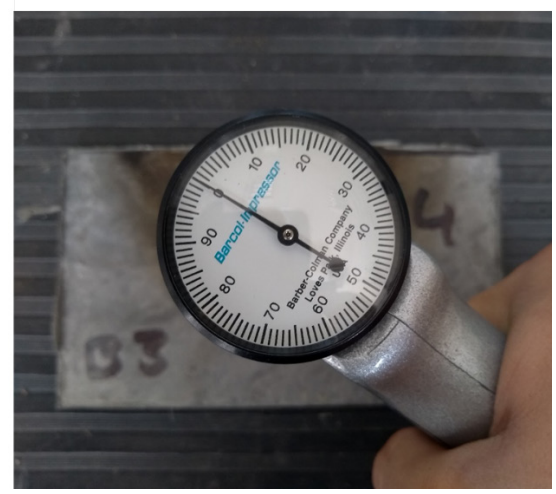

Figura 5. Dureza Barcol (ASTM D2583) de las placas sometidas al ensayo ablativo (llama directa) Fuente: elaboración propia 
En general, se deduce que la incidencia de la llama durante el ensayo ablativo genera una degradación mecánica de los PACMs modificados con polvo cerámico y/o escoria de aluminio de hasta el 23,9\% (30C $+30 \mathrm{E})$, siendo este efecto muy leve $(\leq 6,7 \%)$ para los compuestos fibrorreforzados. Al respecto, Natali et al. [5] y Rodríguez [6] afirman que, en general, las fibras promueven un notable incremento del desempeño mecánico de los PACMs, incluso después de ser sometidos a condiciones superiores a la temperatura de degradación térmica de la matriz (pirólisis de la resina). Con relación a estos hallazgos, cabe agregar que el nivel de degradación de las zonas adyacentes a la incidencia de la llama también depende de la conductividad y la difusividad térmica de los materiales que componen los PACMs.

\subsection{Inspección visual y comportamiento ablativo}

La figura 6 agrupa las imágenes obtenidas durante la inspección visual de la zona térmicamente afectada en las placas sometidas al ensayo ablativo.

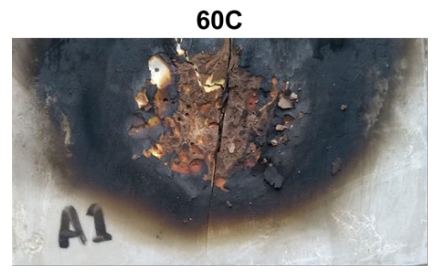

$60 E$

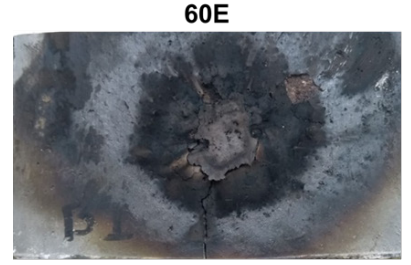

$30 \mathrm{C}+30 \mathrm{E}$

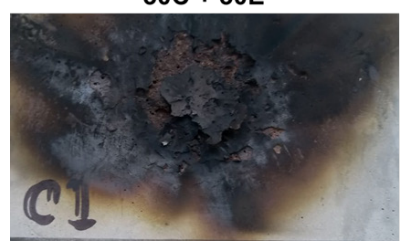

70C

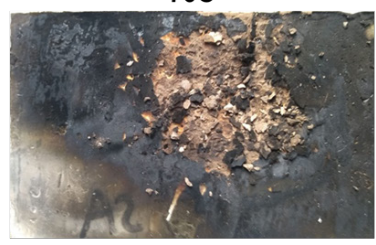

$70 \mathrm{E}$

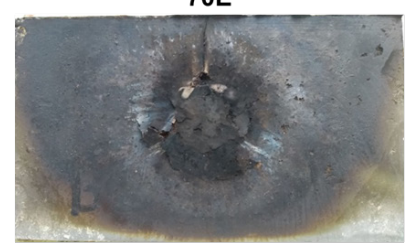

$35 C+35 E$

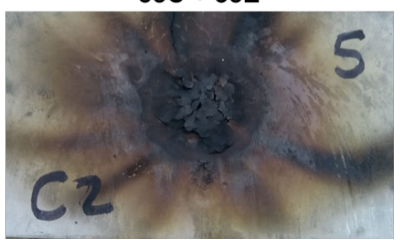

$65 \mathrm{C}+5 \mathrm{~F}$

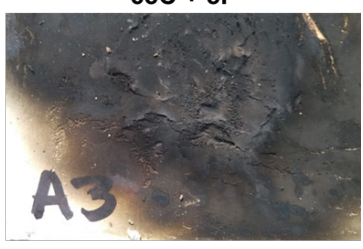

$65 E+5 F$

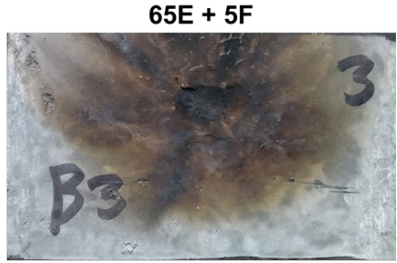

$32,5 \mathrm{C}+32,5 \mathrm{E}+5 \mathrm{~F}$

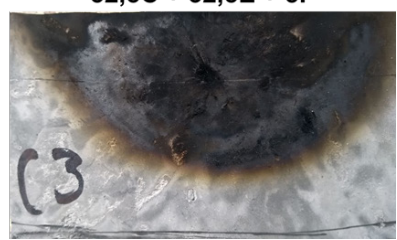

Figura 6. Inspección visual de la zona térmicamente afectada de las placas sometidas al ensayo ablativo

Fuente: elaboración propia

Cuando las placas de PACMs son expuestas al ensayo de llama directa, el calor se transfiere al material ablativo por fenómenos de conducción, convección y radiación. 
Al principio, la temperatura del material aumenta a una velocidad que depende del flujo de calor impuesto y de la energía absorbida por el material. En la superficie, la temperatura aumenta. Cuando el PACM alcanza una temperatura suficientemente alta (generalmente por encima de $\operatorname{los} 200^{\circ} \mathrm{C}$ ), se producen reacciones químicas inducidas térmicamente: la matriz orgánica (resina) sufre un proceso de pirólisis intensiva, lo que genera productos volátiles y una capa carbonizada debido al carbono presente en su estructura y su posterior descomposición térmica [11]. Como resultado de estas reacciones de degradación, una cantidad sustancial de energía es absorbida por los PACMs.

Los gases producidos durante la pirólisis de la matriz polimérica (resina poliéster) quedan atrapados en el material debido a la baja porosidad y la permeabilidad del medio; por lo tanto, la presión interna puede llegar a ser muy alta, lo que lleva a una gran expansión termomecánica de la matriz sólida. Cuando la presión llega a ser suficientemente alta, los gases se difunden a través de la capa carbonizada; en virtud de su presión, los productos de pirólisis forman una capa protectora que actúa como una barrera contra el flujo de calor que impacta directamente al PACM. Esta película de gas también funciona como una barrera de difusión y controla la velocidad de reacción química entre las especies de combustión y la matriz polimérica. Adicionalmente, a medida que la fase gaseosa se filtra a través de la capa porosa del residuo carbonizado, esta absorbe una gran cantidad de calor. Una vez que la región carbonizada transfiere calor a la resina virgen, avanza la pirólisis, de manera que la porosidad y permeabilidad de la capa carbonizada aumentan. Estos fenómenos producen una expansión dimensional en los PACMs, seguido de una fuerte contracción debido al escape de los gases y la reducción gradual de la presión interna, lo cual puede generar fenómenos de erosión o delaminación [5]. De acuerdo con lo reportado por Krzyzak et al. [8], la superficie carbonizada es una capa protectora ante la incidencia directa de la llama, siempre y cuando esta permanezca adherida al PACM. Esta condición se pudo evaluar mediante la inspección visual (figura 6).

Con relación a lo anterior, la figura 6 muestra que el residuo carbonoso permanece en la superficie de los PACMs con escoria de aluminio (60E; 70E; y 65E + 5F), así como en los PACMs con la mezcla de polvo cerámico y escoria de aluminio $(30 \mathrm{C}+$ $30 \mathrm{E} ; 35 \mathrm{C}+35 \mathrm{E}$; y $32,5 \mathrm{C}+32,5 \mathrm{E}+5 \mathrm{~F})$. Por el contrario, los PACMs desarrollados exclusivamente con polvo cerámico $(60 \mathrm{C} \mathrm{y} 70 \mathrm{C})$ presentaron un alto grado de erosión, generación de macro y microgrietas en la zona térmicamente afectada y fenómenos de delaminación del residuo carbonoso (figura 7), lo cual explicaría las diferencias de resistencia a la ablación que se presentaron entre estos compuestos. Mediante la vista transversal de la zona térmicamente afectada (figura 7), se pudo corroborar la formación de un perfil de degradación térmica en función del espesor de la placa de material 
compuesto. Con ello, se destacó que el nivel de deterioro y erosión no alcanza la cara posterior de la placa, lo que demuestra el efecto ablativo de los PACMs desarrollados.

\section{Zona térmicamente afectada}

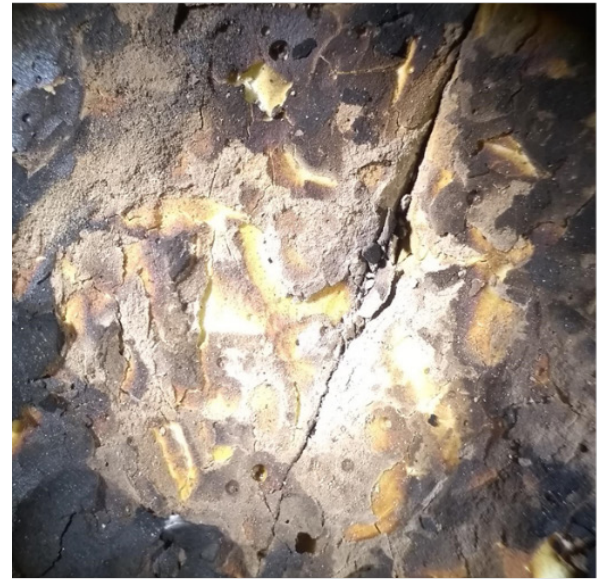

Vista frontal

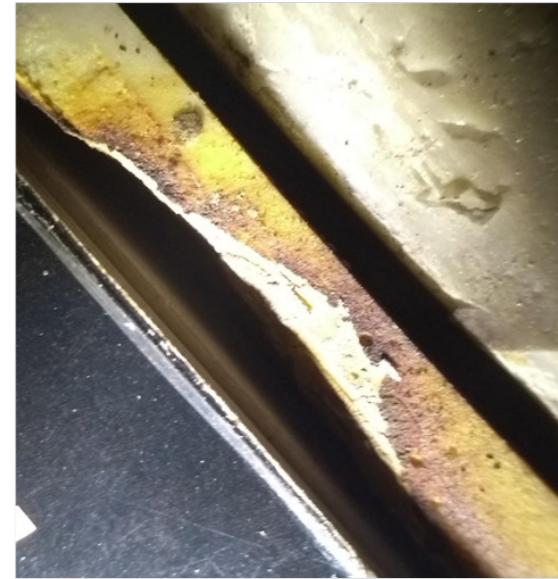

Vista transversal

Figura 7. Observación microscópica de la zona térmicamente afectada (60C):

vista frontal (izquierda) y transversal (derecha)

Fuente: elaboración propia

Un aspecto muy importante por resaltar es que las zonas térmicamente afectadas de todos los PACMs fibrorreforzados $(65 \mathrm{C}+5 \mathrm{~F}$; $65 \mathrm{E}+5 \mathrm{~F}$; y $32,5 \mathrm{C}+32,5 \mathrm{E}+5 \mathrm{~F})$ presentaron una superficie con poca erosión (figura 6), lo cual concuerda con los bajos niveles de pérdida de peso que reportaron estos compuestos (figura 4). Una vez la matriz sufrió el proceso de pirólisis y se convirtió en residuo carbónico, las fibras promovieron la retención del material carbonizado y el proceso de ablación se llevó a cabo de manera efectiva, ya que posiblemente el material pudo continuar absorbiendo calor endotérmicamente. Al respecto, se ha demostrado que cuanto mayor sea la retención de la capa carbonizada, mayor será la eficacia del PACM como disipador de calor o TPS [5]. Complementariamente, la incorporación de la fibra de vidrio redujo los fenómenos de contracción y expansión térmica mencionados anteriormente, así como la posibilidad de que los compuestos presentaran delaminación como consecuencia de la acumulación de los gases atrapados durante la pirólisis y la acumulación de presión en los poros de la matriz. Esto explicaría el aumento de la resistencia a la ablación de los compuestos fibrorreforzados $(65 \mathrm{C}+5 \mathrm{~F} ; 65 \mathrm{E}+5 \mathrm{~F} ; 32,5 \mathrm{C}+32,5 \mathrm{E}+5 \mathrm{~F})$ y su menor tendencia a presentar fisuras. Finalmente, por medio de la inspección visual se corroboró que el mejor desempeño ablativo fue el presentado por el compuesto $65 \mathrm{E}+5 \mathrm{~F}$. 


\section{CONCLUSIONES}

Esta investigación demostró la viabilidad de desarrollar compuestos ablativos de óptimo desempeño utilizando materiales de fácil adquisición y bajo costo. Las materias primas corresponden a una resina poliéster como matriz polimérica y dos tipos de refuerzos: material particulado (60-70 \% en peso) y fibra de vidrio (5\% en peso). Así, se destaca que el particulado puede ser polvo cerámico y/o escoria de aluminio finamente molidos $(<75 \mu \mathrm{m})$, ambos considerados residuos o subproductos industriales.

Los resultados obtenidos son considerados de suma importancia para las industrias de la región relacionadas con los sectores automovilístico, aeronáutico y aeroespacial, así como aquellas industrias que requieran sistemas de protección térmica para los equipos que operan a temperaturas elevadas. Pero estos resultados pueden aportar especialmente a la Fuerza Aérea Colombiana, cuyo propósito de fabricar componentes de propulsión (como los cohetes tipo sonda) con poca dependencia tecnológica externa, se convierte en una posibilidad tangible con este tipo de contribuciones.

El uso de material particulado como modificador de la matriz basada en resina poliéster promovió el desempeño ablativo de los compuestos desarrollados de manera proporcional al porcentaje de incorporación ( $60 \%$ vs. $70 \%$, en peso). Este efecto positivo fue más notorio para el caso de los compuestos modificados con polvo cerámico, en comparación a la escoria de aluminio. Complementariamente, la presencia de fibra de vidrio (5\%) generó un control efectivo de los fenómenos de agrietamiento y delaminación de la capa carbonosa formada por la degradación térmica (pirólisis) de la matriz de resina poliéster, lo que representó un mayor desempeño ablativo de los compuestos fibrorreforzados.

Dentro de los materiales desarrollados, el compuesto modificado con $65 \%$ de escoria de aluminio reforzado con $5 \%$ de fibra de vidrio $(65 \mathrm{E}+5 \mathrm{~F})$ presentó el mejor desempeño ablativo. Alcanzó un nivel de aislamiento térmico frente a la temperatura de la llama incidente $\left(1.600^{\circ} \mathrm{C}\right)$ del $87 \%$, una pérdida de masa posterior al ensayo ablativo del 2,0 \% y una reducción de dureza Barcol de la zona térmicamente afectada del $6,7 \%$. Estos resultados coincidieron con los hallazgos de la inspección visual, en donde el compuesto $65 \mathrm{E}+5 \mathrm{~F}$ mostró el menor grado de erosión sin la presencia de fenómenos de delaminación o agrietamiento de la capa carbonizada.

Los resultados obtenidos promueven el desarrollo de esta línea de investigación en la búsqueda de optimizar los compuestos ablativos con la validación de otros tipos de modificadores y refuerzos de disponibilidad local, así como su aplicación directa en la manufactura de componentes de propulsión como las toberas de los cohetes tipo sonda o de órbita baja, en respuesta a una necesidad especifica de la industria aeroespacial nacional y en especial de la Fuerza Aérea Colombiana. 


\section{AGRADECIMIENTOS}

Los autores, miembros del Grupo de Investigación en Estudios Aeroespaciales (GIEA) de la Escuela Militar de Aviación Marco Fidel Suarez (Emavi), agradecen al Grupo Académico (Gruac), al Programa de Ingeniería Mecánica (Pimec) y a la Sección de Investigación de la Emavi por el apoyo brindado en el marco del proyecto "Facson-1: Desarrollo y construcción de prototipo a escala de cohete sonda con fines cientificos, tecnológicos y militares basado en tecnología colombiana".

\section{REFERENCIAS}

[1] NASA. "Sistema y materiales de protección termica (TPS)," Centro de Investigación Ames, [En línea]. Disponible: https://www.nasa.gov/centers/ames/spanish/research/humaninspace/ humansinspace-thermalprotec.html, 2008.

[2] J. Maldonado Villa, J. Portocarrero Hermann, C. Rodríguez Adaime, J. J. Valbuena Cocunubo y M. E. Acuña Lizarazo, "Evaluación del comportamiento térmico de materiales compuestos de matriz polimérica en prototipos de toberas para cohetes de órbita baja," Rev. Cient. Gen. José María Córdova, vol. 12, n. ${ }^{\circ}$ 13, pp. 275-290, 2014. doi: https://doi. org/10.21830/19006586.163

[3] J. Maldonado Villa, J. Portocarrero Hermann, E. Quitian Barrios, J. Alarcón Orduz y W. David Boada, "Desarrollo de toberas de cohete en material ablativo de matriz polimérica reforzada estructuralmente con fibras de vidrio y carbono," en $71^{\circ}$ Congresso Anual da ABM-Internacional e ao $16^{\circ}$ Enemet - Encontro Nacional de Estudantes de Engenharia Metalúrgica, de Materiais e de Minas, pp. 984-995, 2016.

[4] P. J. Astola, M. A. Rodríguez, F. J. Botana y L. González-Rovira, "Caracterización de elementos de protección térmica de materiales compuestos mediante análisis térmicos," Rev. la Asoc. Española Mater. Compuestos, vol. 2, n. ${ }^{\circ}$ 4, pp. 34-41, 2017.

[5] M. Natali, J. M. Kenny y L. Torre, "Science and technology of polymeric ablative materials for thermal protection systems and propulsion devices : A review," Prog. Mater. Sci., vol. 84, pp. 192-275, 2016. doi: https://doi.org/10.1016/j.pmatsci.2016.08.003

[6] E. S. Rodríguez, "Desarrollo de materiales compuestos avanzados basados en fibras de carbono para la industria aeroespacial," An. la Acad. Nac. Ciencias Exactas, Fis. y Nat., vol. 64, pp. 1-6, 2012.

[7] M. L. Aranzazu Rios, V. P. Muñoz Cárdenas, M. J. Giraldo, Cárdenas, G. H. Gaviria y F. A. González Rojas, "Modelos cinéticos de degradación térmica de polímeros: una revision," Rev. Ing. Univ. Medellin, vol. 12, n. . 23, pp. 113-130, 2013. doi: https://doi.org/10.22395/ rium.v12n23a9

[8] A. Krzyzak, W. Kucharczyk, J. Gaska y R. Szczepaniak, "Ablative test of composites with epoxy resin and expanded perlite," Compos. Struct., vol. 202, , pp. 978-987, 2018. doi: 10.1016/j.compstruct.2018.05.018 
[9] L. Asaro, L. B. Manfredi, S. Pellice, R. Procaccini y E. S. Rodriguez, "Innovative ablative fire resistant composites based on phenolic resins modified with mesoporous silica particles," Polym. Degrad. Stab., vol. 144, pp. 7-16, 2017. doi: 10.1016/j.polymdegradstab.2017.07.023

[10] D. Quiñonez, Y. Lizcano, C. Vaquez, J. Maldonado y J. Portocarrero, “Construcción y evaluación de una tobera a escala menor basada en material compuesto para cohetes de órbita baja,"Rev.inge@UAN, vol. 2,n.o 4, pp. 13-21, 2012.

[11] W. Kucharczyk, D. Dusiński, W. Żurowski y R. Gumiński, "Effect of composition on ablative properties of epoxy composites modified with expanded perlite," Compos. Struct., vol. 183, pp. 654-662, 2018. doi: 10.1016/j.compstruct.2017.08.047

[12] G. Pulci, L. Paglia, V. Genova, C. Bartuli, T. Valente y F. Marra, "Low density ablative materials modified by nanoparticles addition: Manufacturing and characterization," Compos. Part A Appl. Sci. Manuf., vol. 109, pp. 330-337, 2018. doi: 10.1016/j.compositesa.2018.03.025

[13] L. Mohan Kumar, K. M. Usha, E. N. Anandapadmanabhan, and P. Chakravarthy, "Effect of fibre orientation on the properties and functional performance of ablative materials for solid rocket motors," Trans. Indian Inst. Met., vol. 70, n. ${ }^{\circ}$ 9, pp. 2407-2413, 2017. doi: https://doi. org/10.1007/s12666-017-1102-1

[14] ASTM International, "ASTM E285, Standard test method for oxyacetylene ablation testing of thermal insulation materials," 2015.

[15] ASTM International, "ASTM 2583, Standard test method for indentation hardness of rigid plastics by means of a barcol impressor," 2013.

[16] G. P. Suton y O. Biblarz, Rocket Propulsion Elements, $8^{\text {th }}$ ed. New Jersey: John Wiley \& Sons, INC., 2010, 786 p. 